\title{
The use of laryngeal mask airway Supreme ${ }^{\mathrm{TM}}$ in rescue airway situation in the critical care unit
}

Shahla Siddiqui ${ }^{1}$, MD, Edwin Seet ${ }^{1}$, MBBS, MMed, Wing Yan $\underline{\text { Chan }}^{1}$, MBBS

\begin{abstract}
We herein report a witnessed cardiopulmonary collapse of a patient with difficult mask ventilation and nearimpossible laryngoscopy-cum-intubation in the critical care unit. The airway was successfully rescued with a laryngeal mask airway Supreme ${ }^{\mathrm{TM}}$, followed by an open, crash tracheostomy by the otolaryngologist.
\end{abstract}

Keywords: critical care unit, difficult airway, supraglottic airway, tracheostomy

\section{INTRODUCTION}

Airway events with adverse outcomes are more common in critical care units than in the operating rooms, especially those that present during out-of-hours sessions. ${ }^{(1)}$ Supraglottic airway devices play an important role in difficult airway situations. ${ }^{(2)}$ We herein report a witnessed cardiopulmonary collapse of a patient with difficult mask ventilation and anticipated near-impossible tracheal intubation in the critical care unit. The airway was successfully rescued with a laryngeal mask airway Supreme ${ }^{\mathrm{TM}}$, followed by an open, crash tracheostomy by the otolaryngologist.

\section{CASE REPORT}

A 46-year-old, institutionalised man with schizophrenia was diagnosed with oropharyngeal carcinoma two months prior to the acute critical care admission. Magnetic resonance imaging showed an extensive tumour $(5 \mathrm{~cm} \times 3 \mathrm{~cm} \times 6 \mathrm{~cm})$ centred on the right oropharynx extending to the soft palate, right nasopharynx, right vallecula and right extrinsic muscles of the tongue (Fig. 1). There was also bilateral lymphadenopathy. He had received three doses of radiotherapy for the oropharyngeal carcinoma, and the last dose was given on the day of his presenting complaint at the critical care unit. He was referred from the nursing home to the hospital's emergency department with per oral bleeding, wherein excess $1 \mathrm{~L}$ of blood was suctioned from the patient's oral cavity. His haemoglobin level at admission was $7.5 \mathrm{~g} / \mathrm{dL}$. Nasoendoscopy by the otolaryngologist revealed continual oozing from the soft palate tumour and pooling of blood in the hypopharynx. The patient was transferred to the critical care unit for monitoring with plans for angioembolisation of the bleeding tumour. On arrival to the critical care unit at $3 \mathrm{am}$, he was noted to have severe trismus, with a mouth opening of $1.5 \mathrm{~cm}$. The patient was agitated, hypotensive and tachycardic, with a blood pressure of $80 / 61 \mathrm{mmHg}$, heart rate of $135 \mathrm{bpm}$ and oxygen saturation of $76 \%$ on oximetry. Blood transfusion was in progress.

Within minutes, the patient became unresponsive and pulseless. Cardiopulmonary resuscitation commenced; however, mask ventilation was difficult and oxygen saturation failed to improve above $70 \%$ despite administration of $100 \%$ oxygen. The decision was made to insert a Size 3 laryngeal mask airway

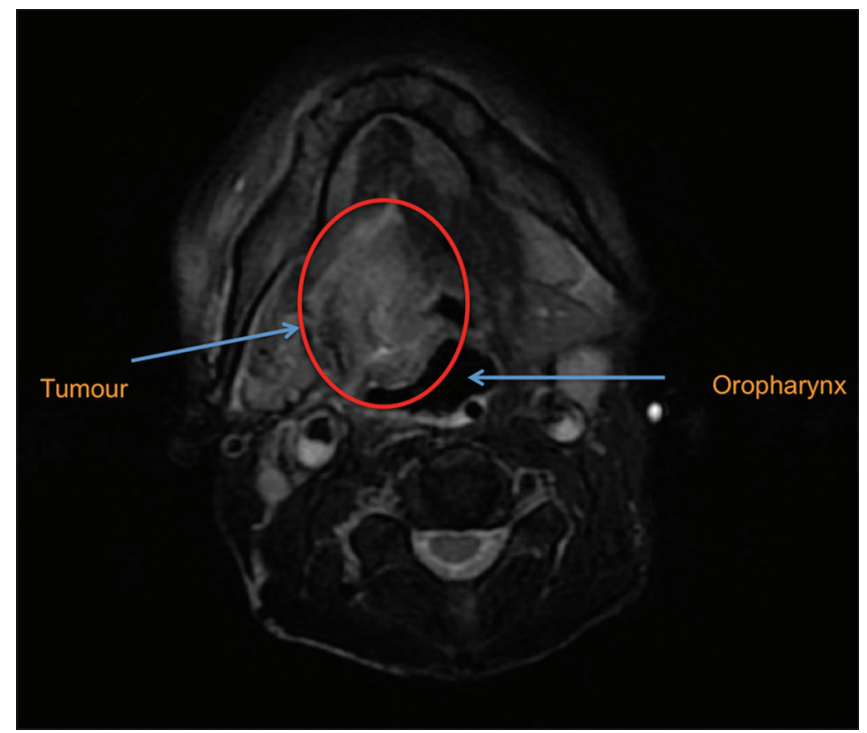

Fig. 1 MR image shows the supraglottic mass.

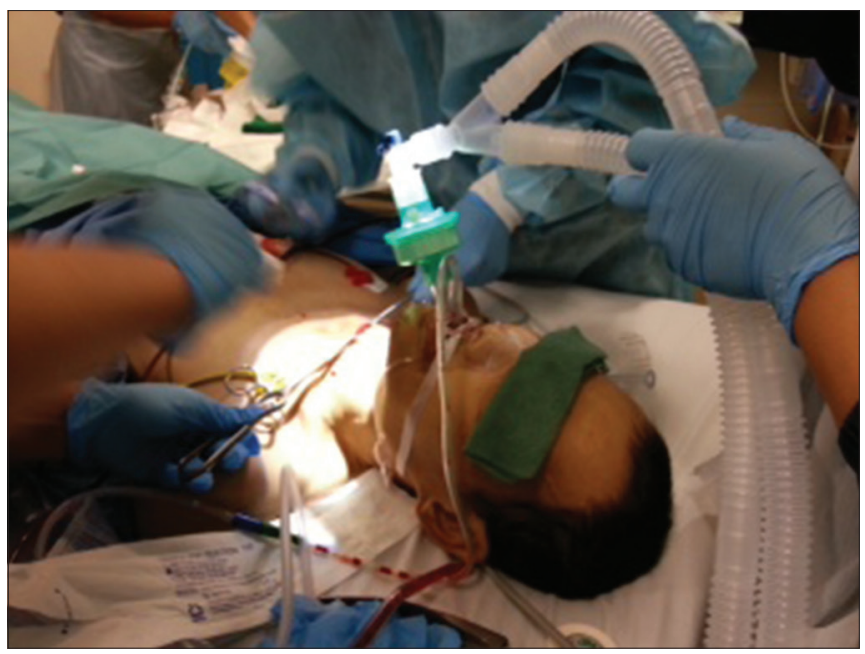

Fig. 2 Photograph shows the patient ventilated via the laryngeal mask airway Supreme ${ }^{T M}$.

Supreme $^{\mathrm{TM}}$ (Fig. 2) with concurrent chest compression and intravenous administration of epinephrine $1 \mathrm{mg}$. Ventilation and oxygenation improved and there was return of spontaneous circulation. The otolaryngologist promptly performed an 
uneventful open tracheostomy via insertion of a Size 6 Shiley ${ }^{\circledR}$ tracheostomy tube (Fig. 2). Bleeding from the soft palate tumour resolved with administration of fresh frozen plasma and tranexamic acid without the need for angioembolisation.

Subsequently, the patient was weaned from sedation and woke up the following day. He tolerated the tracheostomy mask and was discharged from the critical care unit by Day 2 after critical care unit admission. Consent was obtained from the family for the anonymous photograph of the patient.

\section{DISCUSSION}

The Fourth National Audit Project of the Royal College of Anaesthetists and the Difficult Airway Society revealed that airway events in critical care units were more likely than those during anaesthesia, especially in the out-of-hours situation. ${ }^{(1)}$ Postintubation complications are more abundant in the intensive care unit. ${ }^{(3)}$ The outcomes of these cases are particularly adverse. ${ }^{(1)}$ In the recent difficult airway algorithm, supraglottic airway devices were reported to play an important role in inadequate mask ventilation and difficult laryngoscopy with intubation situation. (2) The laryngeal mask airway Supreme ${ }^{\mathrm{TM}}$ was demonstrated to have higher first attempt insertion success in a randomised, controlled trial. ${ }^{(4)}$

In this case report, the collapsed patient had multiple features of a difficult airway in the early morning (3 am), including difficulty in patient cooperation (schizophrenia, agitation, refusal of supplemental oxygen therapy), mask ventilation (due to the recent radiotherapy), and laryngoscopy and intubation (caused by a $1.5 \mathrm{~cm}$ interincisor distance, and bleeding in the oropharynx). Direct laryngoscopy would be near impossible due to the difficulty in inserting a laryngoscope in relation to the small mouth opening. ${ }^{(5)}$ Indirect laryngoscopy with video laryngoscopes (e.g. Glidescope® with a slimmer profile) or rigid optical stylets would be challenging in the face of active oropharyngeal bleeding, obscuring a view necessary for endotracheal tube placement. Similarly, blood and secretions are also likely to obscure visualisation during fibre optic-assisted intubation. Also, fibre optic equipment preparation may take an unacceptably long time in the case of a collapsed patient.

The laryngeal mask airway Supreme ${ }^{\mathrm{TM}}$ has a patented evolution curve and thin profile, which facilitates insertion into a mouth with limited opening. The semi-rigid device allows advancement of the laryngeal mask airway past the oropharyngeal tumour. The inflated cuff rests in the supraglottic area and may prevent further aspiration of blood from the oropharynx. Furthermore, there may be a tamponading effect of the physical laryngeal mask airway on the bleeding of the soft palate tumour. In previous published studies, ${ }^{(4,5)}$ the laryngeal mask airway Supreme ${ }^{\mathrm{TM}}$ has favourable first attempt insertion success of $98 \%$, compared to the ProSeal ${ }^{\circledR}$ laryngeal mask airway. In addition, the laryngeal mask airway Supreme ${ }^{\mathrm{TM}}$ was easily and expeditiously obtained, as it is found in all resuscitation trolleys in our institution, especially during out-of-hours sessions. Little preparation is required except the need for lubrication and a syringe for cuff inflation.

In this case report, the laryngeal mask airway served well in maintaining a patent airway with oximetry saturation of $100 \%$ while the otolaryngologist successfully performed an open tracheostomy. An adverse outcome from this emergency airway scenario was averted and the patient was both successfully resuscitated and discharged from the critical care unit within two days.

In conclusion, in the latest difficult airway algorithm promulgated by the American Society of Anesthesiologist, the supraglottic airway device plays an expanded role in difficult airway management, especially for failed mask ventilation and laryngoscopy and intubation. ${ }^{(6)}$ We have herein reported a successful resuscitation of a collapsed patient with a difficult airway via a laryngeal mask airway Supreme ${ }^{\mathrm{TM}}$, highlighting the peculiar advantageous features of this supraglottic airway device.

\section{REFERENCES}

1. Cook TM, Woodall N, Harper J, Benger J. Fourth National Audit Project. Major complications of airway management in the UK: results of the Fourth National Audit Project of the Royal College of Anaesthetists and the Difficult Airway Society. Part 2: intensive care and emergency departments. Br J Anaesth 2011; 106:632-42.

2. Apfelbaum JL, Hagberg CA, Caplan RA, et al. American Society of Anesthesiologists Task Force on Management of the Difficult Airway. Practice guidelines for management of the difficult airway: an updated report by the American Society of Anesthesiologists Task Force on Management of the Difficult Airway. Anesthesiology 2013; 118:251-70.

3. Jaber S, Amraoui J, Lefrant JY, et al. Clinical practice and risk factors for immediate complications of endotracheal intubation in the intensive care unit: a prospective, multiple-center study. Crit Care Med 2006; 34:2355-61.

4. Seet E, Rajeev S, Firoz T, et al. Safety and efficacy of laryngeal mask airway Supreme versus laryngeal mask airway ProSeal: a randomized controlled trial. Eur J Anaesthesiol 2010; 27:602-7.

5. Lavery GG, McCloskey BV. The difficult airway in adult critical care. Crit Care Med 2008; 36:2163-73.

6. Lim MS, Hunt-Smith J. Difficult airway management in the intensive care unit: practical guidelines. Crit Care Resusc 2003; 5:43-52. 